\title{
Trends and developments in intra-day margining and same-day settlement
}

\author{
Frances Maguire
}

E-mail: fr@nces.fslife.co.uk

Received: 5th April, 2005

Frances Maguire is a freelance journalist with more than ten years' writing experience, specialising in topics such as derivatives, risk management, banking IT, securities processing and fund management. She was previously Associate Editor on Futures \& Options World, Editor of Futures \& Options Week and Banking Technology and technology writer for Financial News.

\begin{abstract}
LCH. Clearnet believes a daily intra-day margin call will bring greater security to the European exchange-traded derivatives market, but it brings with it the pressure to move to same-day settlement. The author assesses the impact this will have and asks whether the risk mitigation benefits outweigh the cost and upheaval for clearing firms.
\end{abstract}

\section{INTRODUCTION}

LCH.Clearnet will shortly introduce a daily intra-day margining policy for

exchange-traded futures and options, using its new Intra-day Risk Information System (IDRiS) margining tool. The new policy is based on full revaluation and recalculation of initial margin requirements in respect of all registered contracts, including contracts entered into on the day of the call.

The policy provides for a routine call to clearing members in the middle of the day to collect additional margin liabilities above a de minimis value of $\mathcal{E} 10,000$ (US $\$ 18,500$ ). Currently, LCH.Clearnet calls intra-day margins for everything but futures and options, and SwapClear and RepoClear, now in their sixth year, have provided models for IDRiS best practice. Andrew White, director of risk policy at LCH.Clearnet, says: 'Similar to the end of day margin call, we will take positions and prices real time and then re-calculate profit and loss and initial margins using standard portfolio analysis of risk (SPAN). The difference between IDRiS, and RepoClear and SwapClear margining systems, is that in futures and options business, position-keeping is done with systems that are unique to the exchanges, ie a different system for each exchange, so IDRiS has to interface with a number of systems.' IDRiS takes feeds directly from trade registration system/clearing processing system (TRS/CPS) for Liffe and the Inter Continental Exchange/International Petroleum Exchange (ICE/IPE), and from LME Matching and Clearing for the London Metal Exchange.

According to White, intra-day margining will enable the clearing house to take risk out of the market. He says: 'What it enables us to do is to register the futures
Derivatives Use, Trading \& Regulation, Vol. 11 No. 1, 2005, pp. 10-15 (C) Henry Stewart Publications, $1747-4426$ 
and options contracts intra-day. There is currently a window of risk, however theoretical, before we register contracts at the end of the day. Here we are talking about real-time intra-day registration of futures and options, which we would regard as best practice.'

At noon every day, a snapshot of positions will be taken as an indicative run to give members an idea of what would be called at $1 \mathrm{pm}$, were nothing to change. If a clearing member has significant give-up business at midday, under the exchange rules, they should be claiming or giving them up within half an hour, and they then have one hour before the real margin call occurs. But White adds: 'If you do a trade at 12.59, and you are going to give that up, you are going to get captured at 13.00 by the intra-day call. This is just the nature of taking a real-time snapshot of positions.'

\section{SECURING OVERNIGHT RISK}

Patrick Cirier, London-based Head of Operations at international broker and clearer Fimat, says: 'We need the clearing house to be safe and secure. There is a risk there that waiting for a night margin call may not cover 100 per cent of exposure. We are clearing in real time - matching the trades, receiving client confirms, giving-up trades out and taking up trades given to us in real time. What we will put in place, once we have the precise IDRiS role, are the prices that we will run our margining processing on.'

Fimat gives up and takes up several hundreds of credit lines daily and already has real time trade capture and the tools for real time give-ups and take-ups. In preparation for the introduction of intra-day margining, Fimat is ensuring that its clearing clients are ready and have the mechanism in place for same-day payments. But Cirier agrees that the intra-day margin call will have an impact on give-ups. 'Clearing for large execution parties will come under scrutiny. If there is any delay in the give-up process you can end up paying margins for a lot of give-up trades, which you will get rid of minutes after the margin-call so it will skew the large execution firms which are doing more execution than clearing.'

According to LCH.Clearnet's own research with its members, the impact of a second margin call will not be a great as might be expected. White adds: 'We discussed this with a working group on IDRiS, made up of a cross-section of members, using a month's worth of historic data. Obviously, if a member with relatively small margins does a very large trade that they are giving up, and they do it at 12.59, then the size of the call will reflect this, but this is not what we saw happen most of the time through a month's worth of members' data. We have found that the offsets significantly reduce the size of the margin calls.'

Futures and options contracts cleared by LCH.Clearnet are already subject to margin calls on an almost daily basis. But, as current intra-day calls from the clearing house are on a contract by contract basis, there are no offsets between contracts, whereas IDRiS will be a portfolio-based calculation allowing offsets. 'So individual members used to seeing an intra-day 
margin call, say for the FTSE at a certain time during the day, then on Euribor at a certain time of the day, and equity options at a different time again, will see one call, for one figure,' says White.

Originally slated for launch in Q1 of 2004, the IDRiS system — which was already in development prior to LCH's merger with Clearnet - is still being tested internally. Members will shortly be given a month's notice before the commencement of full end-to-end member testing, which will then run for another month. According to White, this live demo will provide members with daily intra-day reports, so that they can get idea of what their intra-day calls will look like.

\section{ONE MARGIN CALL FOR ALL MARKETS}

At the moment, IDRiS will operate for futures and options only, but LCH.Clearnet is planning to move eventually to one call across all markets. White says: "We would need to do quite a bit of work with our members to do this because at the moment they are used to handling reconciliation on each individual system.'

Moving away from next-day settlement to same day brings with it a range of problems, not least that few banks have back offices that could conduct a full reconciliation process within the timeframe given - one hour - to pay the margins in order to recalculate and call customer margins. While the intra-day margin call will hopefully make firms give up trades for clearing on a more timely basis, the problem with mismatches still remains.
While members will not be called for margins on a mismatched trade - these trades are flagged and ignored by IDRiS it means that the risk remains with the member, and the need to manage the process remains even greater, as these trades will not be registered and novated to the clearing house. White adds: 'All information will be made available at $1 \mathrm{pm}$ daily, in terms of settlement prices, positions and SPAN risk arrays. It is down to the individual clearing member as to whether they want to take that into their systems intra-day or not, whether they actually want to fully reconcile or just use the reporting that we will make available in terms of the margin numbers and where those margin numbers are coming from.'

LCH.Clearnet will not pay out any intra-day profits (ie profits from mark-to-market movements) to firms. Rather, it will net intra-day profits against increases in initial margin, thus reducing the size of the potential intra-day call. In the US, both the Common Clearing Link for Chicago and Mercantile Exchange and Chicago Board of Trade and The Clearing Corporation (CCorp), pay out intra-day as well as collect margins.

Kim Taylor, Managing Director and President of the Clearing House Division, Chicago Mercantile Exchange, says it began intra-day mark-to-market margining on the day of the 1987 stock market crash and, in 1992, with the launch of its overnight trading system, Globex, began doing a routine calculation and collection of initial margins at $1 \mathrm{pm}$, when it began both collecting and paying out margins, intra-day. 
Taylor says: 'In order for a clearing house to be comfortable paying out at intra-day they need to feel comfortable with the intra-day risk management protections and the solidity of the process they are using at intra-day. We are using the identical process intra-day as we are doing at night - doing the same things in terms of finalising positions, having the firms report to us their positions, and using the same offsets between contracts as recognised end of day.

'From a risk management point of view it has had a very positive effect because it enables us to remove accumulated debt obligations from the system sooner and also enables us to clear and collect margins on new positions sooner. As more trading happens around the clock it is important for a clearing house to clear and margin those positions sooner.'

Using a slightly different intra-day model, CCorp - which clears Eurex US calculates both variation and initial margin on a daily intra-day basis, at $1 \mathrm{pm} \mathrm{CST,}$ but only variation margin is fully paid in the 'midday' cycle. Although, unlike the LCH.Clearnet model, CCorp does not wait until the end of day margin call to pay out to member firms, 10 per cent of all 'collects' (where a firm has a profit from a mark-to-market position) are held back at the midday call. Any original midday margin deficits are also held back in collects situations. 'This midday variation margin cycle mitigates the majority of the overnight settlement risk in the marketplace by CCorp collecting on average 80 per cent of all losses on a top day basis,' says Chip Chong, director of risk services at CCorp.
The fact that CCorp does not collect initial margins intra-day has made it possible for clearing firms to manage the margin call without necessarily running a full reconciliation process at midday and continuing to collect initial margins from customers once a day. The Clearing Corp hosts a web-enabled risk tool that allows clearing participants and CCorp risk management staff to monitor variation margins on a real time basis and original margin calculations on a 30-minute basis. Chong adds: 'This web risk tool allows our clearing participants to monitor their market risk at anytime on a global basis.'

Until now, the derivatives markets have operated in a $T+1$ environment, where customer margins are collected by the banks the morning after trade, enabling banks to run full reconciliation and batch processing in their back offices overnight. The introduction of a routine margin call could effectively push the industry to same-day settlement, if banks make a margin call on their customers intra-day, rather than pay the margins on their behalf, or simply increase initial margins to try and cover the midday call. But there are competitive issues at stake, and the jury is still out on whether banks will cover or call, or come up with an alternative arrangement with their customers.

\section{PASSING ON THE COST}

Jos Schmidt, consultant with Capco, a global financial services consultancy firm, says that while the move to intra-day margining by a clearing house is a logical evolution of a market growing in size and volatility, the 
implications for clearing firms are far reaching. He says: 'A routine daily margin call will have to be passed on to customers. It simply is not sustainable otherwise. But the complexity it causes from a back office perspective is significant. Some banks would be able to process intra-day margin calls, but many are unable.'

Even if clearing firms do decide to pass on the margin call to their customers, it is unclear how this would work within the timeframe proposed. In order to conduct the full reconciliation process, the files would need to be downloaded from the clearing house and matched to the same cut-off period with the firm's back office reconciliation system. According to SunGard Futures Systems' Managing Director in London, this process could take up to two hours. Then it is only possible with customers that can conduct immediate electronic payment. Clearing firms have one hour after the margin call to make the payments to the Protected Payment System.

Patrick Thornton-Smith, Managing Director of MarginClick, believes the move to intra-day margining is long overdue. $\mathrm{He}$ says: 'This is a big issue. Without intra-day margining there is exposure to major events that occur during trading hours. The fact the back office is still on next day settlement, while the front office is in real time, is something that needs to be addressed in any case, in order for the industry to achieve true straight through processing.'

\section{PAIN WITHOUT GAIN?}

Conversely, Alex Wilkinson, head of listed products at Dresdner Kleinwort Wasserstein in London, believes that intra-day margin calls should remain on a case-by-case basis. He says: 'The holistic purpose of intra-day margining is to reduce the amount of systemic risk within the financial markets. It does not do this due to a number of operational reasons. If a clearing house makes an intra-day call, the likelihood of a futures commissions merchant (FCM) passing on that call onto a customer is zero.'

The reason for this is that setting-up intra-day payments is complex and unusual. If the result of the calculation is that the clearing house owes money, it is also unable to pay it until the next day. The net result is that clearing members are funding the payables overnight and the difference between the receivables overnight. If the purpose is to make the clearing house fiscally safer, it does succeed to a certain extent, but it increases the cost of doing business, and there is a marginal increase in the operational risk without necessarily making the FCM community any more fiscally resilient.

Says Wilkinson: 'There is a more effective way of managing intra-day volatility which is already available to clearinghouses. They have the ability at any point in time to call supra-margins. They can call additional funds or collateral from member firms in the event that they feel uncomfortable either by the institution's credit rating taking a sharp fall or that the volatility in the marketplace around their positions is such that on a perceived VAR-basis that VAR number increases substantially. A cost effective way of managing those issues would be for the 
clearinghouse to exercise, as it has done in the past, its supra-margining ability.'

Instead of deferring the responsibility and the cost to members, the clearing house would have to manage that risk and be seen to do so in a fair though subjective manner, and if the clearing house makes the wrong call (ie not calling sufficient funds), it risks the regulator calling its ability into question. The introduction of a second daily margin call calls into question the role of a clearing house: it is perceived to be there to take risk out of the market by providing a central counterparty but, in reality, a clearing house is aiming to be seen as zero risk. In 2000, continuous linked settlement (CLS) was launched to take settlement risk out of the foreign exchange markets. This model differs slightly from a central counterparty in that CLS does not take the other side of every deal but, instead, simply offers simultaneous exchange of each leg of a foreign exchange deal.

'This last step towards same-day settlement looks very like 'continuous linked settlement', says Wilkinson, 'but if you have continuous linked settlement why do you need a clearing house? If financial futures traded throughout the day are debited and credited automatically, so that theoretical credit and market risks were obviated, in real time, and that Herstatt risk was the last risk left to be settled, that changes the role and the shape of the clearing houses quite dramatically. That transition opens up their current business models to significant competition.'

There is no doubt that a substantial proportion of the exposure held by the clearing house will be mitigated by an intra-day margin call on the futures and options market. White, at LCH.Clearnet, says: 'It is in the members' interests that the absolute minimum risk is actually carried by the clearing house at any one time. Having this new software in place will make for a better and more secure counterparty and that must serve the interests of the market and its users.'

But is this extra security needed? And where does it end? Why just one or two margin calls daily, why not hourly margin calls, or in real time? At what point does the benefit begin to outweigh the cost? Most clearing firms, such as Fimat, are already monitoring risk in real time, which can lead to the clearer stopping a customer from building up a large position but, for Cirier, this in no way means that margining should ever be in real time. He says: 'Further daily margin calls would simply not be feasible. You need to have a cut-off time where positions are identified and where you have a reference price you can work with.' 